\title{
Chezy's Resistance Coefficient in a Circular Conduit
}

\author{
Bachir Achour*
}

Research Laboratory in Subterranean and Surface Hydraulics University of Biskra, PO Box 145 RP, 07000, Biskra, Algeria

\begin{abstract}
In the literature, there is no explicit method for calculating the resistance coefficient of Chezy, especially for a circular conduit. Existing relationships are either implicit or do not take into account all parameters influencing the flow such as kinematic viscosity or the slope of the conduit. In many practical cases, one affects arbitrarily a constant value for Chezy's coefficient. It is a physically unjustified approach, because Chezy's coefficient varies with flow parameters, especially the filling rate of the conduit and the absolute roughness. In this paper, simple and explicit relationships are presented for the calculation of Chezy's resistance coefficient in a circular conduit. These relationships have been established based on the rough model method. The Chezy's resistance coefficient is expressed in terms of known hydraulic parameters of the flow in a referential rough model. For fast calculation of Chezy's coefficient, the simplified method is the most appropriate since it requires only four parameters which are the discharge, the absolute roughness, the slope and the kinematic viscosity. The study also shows that the Chezy's resistance coefficient reaches a maximum whose expression is well defined. Some examples are presented showing how to calculate Chezy's coefficient in a circular conduit with a minimum practical data.
\end{abstract}

Keywords: Chezy's coefficient, circular conduit, discharge, energy slope, hydraulic radius, rough model method.

\section{INTRODUCTION}

Referring to the literature, we can see that few formulae exist for expressing Chezy's resistance coefficient $C$. The most frequently cited are the old formulae of GuanguilletKutter [1], Bazin [2] and Powell [3]. These relationships are well summarized and discussed by Chow [4].

The Guanguillet-Kutter formula expresses $C$ in terms of the hydraulic radius $R_{h}$, the coefficient of roughness $n$ known as Kutter's $n$ and the slope $S$. In English units, this formula is:

$$
C=\frac{41.65+\frac{0.00281}{S}+\frac{1.811}{n}}{1+\left(41.65+\frac{0.00281}{S}\right) \frac{n}{\sqrt{R_{h}}}}
$$

This relationship does not contain a term relating to the kinematic viscosity. Thus, it can not be applied to the entire domain of turbulent flow. Its application seems to be restricted to the rough domain for which the kinematic viscosity has no effect.

Bazin formula expresses the coefficient $C$ as a function of hydraulic radius $R_{h}$, but not of the slope $S$. This formula is:

*Address correspondence to this author at the Research Laboratory in Subterranean and Surface Hydraulics University of Biskra, PO Box 145 RP, 07000, Biskra, Algeria; Tel: 0021333522315; Fax: 0021333522315;

E-mail: bachir.achour@larhyss.net

$$
C=\frac{87}{1+\frac{m}{\sqrt{R_{h}}}}
$$

Where $m$ is a coefficient of roughness whose values are given by a table as a function of the type of the material forming the channel or the conduit. As for the GuanguilletKutter formula, Bazin formula contains no terms of kinematic viscosity. It does not therefore apply to the whole domain of turbulent flow.

The Powell formula is more complete as it contains the hydraulic radius $R_{h}$, the absolute roughness $\varepsilon$ and the Reynolds number $R_{\mathrm{e}}$. However, this formula is implicit, expressing $C$ as:

$$
C=-42 \log \left(\frac{C}{4 R_{e}}+\frac{\varepsilon}{R_{h}}\right)
$$

According to this relationship, $C$ depends especially on the Reynolds number $R_{\mathrm{e}}$ and therefore on the kinematic viscosity $v$. In this relation, there is no term that expresses the influence of the slope $S$ on the coefficient $C$. Its application seems to be suitable for the entire domain of turbulent flow. It is interesting to note that Powell formula contains the absolute roughness $\varepsilon$ which is a measurable parameter in practice. To determine the coefficient $C$ by the Powell formula, it is necessary to use a trial-and-error procedure.

More recently, Swamee and Rathie [5] have attempted to propose a general relationship for Chezy's coefficient $C$, 
applicable in the entire domain of turbulent flow and for all shapes of channels and conduits. However, this relationship is implicit, requiring also a trial-and-error procedure especially when the linear dimension of the channel or conduit is not given, or when it comes to compute the normal depth of the flow. Swamee and Rathie suggested for $C$ a logarithmic formula as:

$$
C=-2.457 \sqrt{g} \ln \left(\frac{\varepsilon}{12 R_{h}}+\frac{0.221 v}{R_{h} \sqrt{g S R_{h}}}\right)
$$

Apart from its implicit form, this relationship has the advantage of being very complete. All the flow parameters are included in this relationship.

According to the literature, several tests were performed on corrugated pipes or large scale roughness in channels of non circular cross section that have not led to a convincing formula for Chezy's coefficient.

Among these studies, we can mention those of Streeter [6], Ead and al. [7], Pyle and Novak [8], Marone [9], Perry and al. [10], Naot and al. [11]. More recently, Giustolisi [12] used a genetic programming to determine Chezy's resistance coefficient for full circular corrugated channels. For commercial pipes or artificial channels, the literature does not indicate specific studies. That is why this article is proposed which aims to establish simple relationships for calculating Chezy's coefficient based on practical data. The calculation approach is based on the rough model method (RMM) that has been proven in the recent past by contributing successfully to the design of conduits and channels and to the calculation of normal depth [13-25]. Two explicit methods of calculating Chezy's coefficient are proposed. The first method considers the filling rate of the conduit, while the second one is more simplified. It takes no account of the filling rate of the conduit or its diameter. Both methods give similar results. In this article, examples are provided to better appreciate the ease of the method and calculation.

\section{HYDRAULIC PROPERTIES}

The characteristics of the flow in a circular conduit partially occupied (Fig. 1) are, in particular:

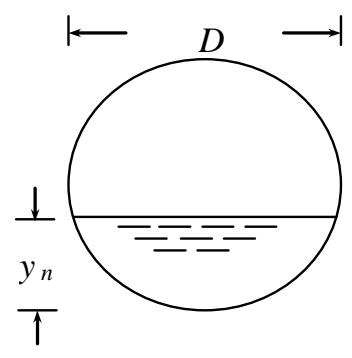

Fig. (1). Flow in a circular conduit.

1. The wetted area:

$A=\frac{D^{2}}{4}\left[\cos ^{-1}(1-2 \eta)-2(1-2 \eta) \sqrt{\eta(1-\eta)}\right]$
It thus appears that the wetted area is depending on the diameter $D$ of the conduit and the filling rate $\eta=y_{n} / D$, where $y_{n}$ is the normal depth.

Eq. (5) can be written as:

$A=\frac{D^{2}}{4} \sigma(\eta) \varphi(\eta)$

where:

$\sigma(\eta)=\cos ^{-1}(1-2 \eta)$

$\varphi(\eta)=1-\frac{2(1-2 \eta) \sqrt{\eta(1-\eta)}}{\cos ^{-1}(1-2 \eta)}$

For a circular conduit completely filled, corresponding to $\eta=1$, one can deduce from Eq. (7) and Eq. (8) respectively that $\sigma(\eta=1)=\pi$ and $\varphi(\eta=1)=1$.

2. The Wetted perimeter

$P=D \cos ^{-1}(1-2 \eta)$

This can be written simply as:

$P=D \sigma(\eta)$

3. The hydraulic radius $R_{h}=A / P$ is thus:

$R_{h}=\frac{D}{4} \varphi(\eta)$

\section{GENERAL RELATIONSHIP OF CHEZY'S RESIS- TANCE COEFFICIENT}

Chezy's relation gives the discharge $Q$ as:

$Q=C A \sqrt{R_{h} S}$

To highlight the variation of Chezy's coefficient based on all parameters governing the flow, Achour and Bedjaoui formula [23] is very useful. This relationship, applicable to all geometric profiles, was established in the whole domain of turbulent flow encompassing smooth, transition and rough regimes. According to Achour and Bedjaoui [23], the discharge $Q$ is given by the following formula:

$Q=-4 \sqrt{2 g} A \sqrt{R_{h} S} \log \left(\frac{\varepsilon}{14.8 R_{h}}+\frac{10.04}{R_{e}}\right)$

where $S$ is the slope of the conduit, $R_{\mathrm{e}}$ is a Reynolds number, $\varepsilon$ is the absolute roughness and $g$ is the acceleration due to gravity. The Reynolds number $R_{\mathrm{e}}$ is governed by the following equation:

$R_{e}=32 \sqrt{2} \frac{\sqrt{g S R_{h}^{3}}}{v}$

where $v$ is the kinematic viscosity. 
Inserting Eq. (11) into Eq. (14), leads to:

$$
R_{e}=4 \sqrt{2} \frac{\sqrt{g S D^{3}}}{v}[\varphi(\eta)]^{3 / 2}
$$

For a circular conduit completely filled, the hydraulic radius is $R_{h}=D / 4$. Thus, Eq. (14) becomes:

$R_{e f}=4 \sqrt{2} \frac{\sqrt{g S D^{3}}}{v}$

The subscript " $f$ " refers to the full state of the conduit. Taking into account Eq. (16), Eq. (15) can be rewritten as:

$R_{e}=R_{e f}[\varphi(\eta)]^{3 / 2}$

Comparing Eq. (12) and Eq. (13), it is obvious that Chezy's coefficient is such that:

$C=-4 \sqrt{2 g} \log \left(\frac{\varepsilon}{14.8 R_{h}}+\frac{10.04}{R_{e}}\right)$

or, in dimensionless form :

$\frac{C}{\sqrt{g}}=-4 \sqrt{2} \log \left(\frac{\varepsilon}{14.8 R_{h}}+\frac{10.04}{R_{e}}\right)$

Inserting Eq. (11) and Eq. (17) into Eq. (19) leads to:

$\frac{C}{\sqrt{g}}=-4 \sqrt{2} \log \left(\frac{\varepsilon / D}{3.7 \varphi(\eta)}+\frac{10.04}{R_{e f}[\varphi(\eta)]^{3 / 2}}\right)$

It thus appears that $C$ depends on the relative roughness $\varepsilon / D$, the filling rate $\eta$ and the Reynolds number $R_{e f}$. When these parameters are given, relation (20) allows the explicit determination of the coefficient $C$. However, when it comes to design the conduit, $D$ is not a given data and only $Q, \eta, S, \varepsilon$ and $v$ are the known parameters. In this case, Eq. (20) does not allow determining explicitly the coefficient $C$. However, this problem can be solved using the rough model method (RMM).

\section{COMPUTATION OF CHEZY'S RESISTANCE CO- EFFICIENT}

\subsection{The Rough Model Method}

The rough model is a circular conduit of diameter $\bar{D}$ greater than $D$, in which the flow is characterized by a friction factor $\bar{f}=1 / 16$, arbitrarily chosen. This high friction factor implies that the flow in the model is rough. In the rough model, the discharge is $Q$, the slope is $S$, the kinematic viscosity is $v$, and the filling rate is $\boldsymbol{\eta}$. Taking into account that Chezy's resistance coefficient in the rough model is $\bar{C}=\sqrt{8 g / \bar{f}}$, one may write:
$\bar{C}=8 \sqrt{2 g}=$ constant

According to the RMM [13, 24, 25], $D$ and $\bar{D}$ are related by the following equation:

$D=\psi \bar{D}$

where $\psi$ is a non-dimensional correction factor of linear dimension, less than unity. It was demonstrated that $\psi$ can be written as:

$\psi=(16 f)^{1 / 5}$

$\psi=1.35\left[-\log \left(\frac{\varepsilon / \overline{R_{h}}}{19}+\frac{8.5}{\overline{R_{e}}}\right)\right]^{-2 / 5}$

where $\bar{R}_{h}$ and $\bar{R}_{e}$ are respectively the hydraulic radius and the Reynolds number in the rough model. The Chezy's resistance coefficient $C$ and the friction factor $f$ are as $C=\sqrt{8 g / f}$. As a result, Eq. (23) leads to:

$C=\frac{8 \sqrt{2 g}}{\psi^{5 / 2}}$

which can be rewritten as:

$C=\frac{\bar{C}}{\psi^{5 / 2}}$

Inserting Eq. (11) and Eq. (17) into Eq. (24), leads to:

$\psi=1.35\left[-\log \left(\frac{\varepsilon / \bar{D}}{4.75 \varphi(\eta)}+\frac{8.5}{\overline{R_{e f}}[\varphi(\eta)]^{3 / 2}}\right)\right]^{-2 / 5}$

Combining Eq. (25) and Eq. (27), one can write:

$C=-5.343 \sqrt{g} \log \left(\frac{\varepsilon / \bar{D}}{4.75 \varphi(\eta)}+\frac{8.5}{\overline{R_{e f}}[\varphi(\eta)]^{3 / 2}}\right)$

The Reynolds number $\overline{R_{e f}}$ is given by Eq. (16) as:

$\overline{R_{e f}}=4 \sqrt{2} \frac{\sqrt{g S \bar{D}^{3}}}{v}$

Eq. (28) will be used when the diameter $D$ of the conduit is not a given data of the problem. The coefficient $C$ is explicitly calculated provided the discharge $Q$, the slope $S$, the absolute roughness $\varepsilon$ and the filling rate $\eta$ are given. To express the diameter $\bar{D}$, apply Chezy's relation to the rough model. Hence: 


$$
Q=\bar{C} A \sqrt{\overline{R_{h}} S}
$$

Taking into account Eq. (6), Eq. (11) and Eq. (21), Eq. (30) leads to:

$$
\bar{D}=[\sqrt{2} \sigma(\eta)]^{-0.4}[\varphi(\eta)]^{-0.6}\left(\frac{Q}{\sqrt{g S}}\right)^{0.4}
$$

Eq. (31) permits a direct determination of the diameter $\bar{D}$, since $Q, S, \eta$ and $g$ are the known parameters of the problem. Thus, all relationships are established for the explicit determination of the Chezy's coefficient $C$, through the following steps provided $Q, S, \boldsymbol{\eta}, g$ and $\boldsymbol{v}$ are given:

1. For the given value of the filling rate $\eta$, compute $\sigma(\eta)$ and $\varphi(\eta)$ according to Eq. (7) and Eq. (8) respectively.

2. For the given values of $Q, S, \eta$ and $g$, compute the diameter $\bar{D}$ of the rough model by applying Eq. (31).

3. For the given values of $\bar{D}, S, g$ and $v$, Eq. (29) gives the Reynolds number $\overline{R_{e f}}$.

4. Finally, using Eq. (28), Chezy's resistance coefficient $C$ is worked out for the known values of $\eta, \varepsilon, \bar{D}$, $\overline{R_{e f}}$ and $g$.

\subsection{Example 1}

For the following data, compute Chezy's resistance coefficient:

$Q=0.987 \mathrm{~m}^{3} / \mathrm{s}, \quad S=3 \times 10^{-4}, \quad \varepsilon=10^{-4} \mathrm{~m}, \quad \eta=0.6$, $v=10^{-6} \mathrm{~m}^{2} / \mathrm{s}$.

1. According to Eq. (7) and Eq. (8), $\sigma(\eta)$ and $\varphi(\eta)$ are respectively:

$\sigma(\eta)=\cos ^{-1}(1-2 \eta)=\cos ^{-1}(1-2 \times 0.6)$

$=1.77215425$

$\varphi(\eta)=1-\frac{2(1-2 \eta) \sqrt{\eta(1-\eta)}}{\cos ^{-1}(1-2 \eta)}$

$=1-\frac{2 \times(1-2 \times 0.6) \times \sqrt{0.6 \times(1-0.6)}}{\cos ^{-1}(1-2 \times 0.6)}$

$=1.110576819$

2. In accordance with the relationship (31), the diameter $\bar{D}$ of the rough model is:

$$
\bar{D}=[\sqrt{2} \sigma(\eta)]^{-0.4}[\varphi(\eta)]^{-0.6}\left(\frac{Q}{\sqrt{g S}}\right)^{0,4}
$$

$$
\begin{aligned}
& =[\sqrt{2} \times 1.77215425]^{-0,4} \times 1.110576819^{-0.6} \\
& \times\left(\frac{0.987}{\sqrt{9.81 \times 3 \times 10^{-4}}}\right)^{0.4}=2.075071373 \mathrm{~m}
\end{aligned}
$$

3. Applying Eq. (29), the Reynolds number $\overline{R_{e f}}$ is then :

$$
\begin{aligned}
& \bar{R}_{e f}=4 \sqrt{2} \frac{\sqrt{g S \bar{D}^{-3}}}{v} \\
& =4 \times \sqrt{2} \times \frac{\sqrt{9.81 \times 3 \times 10^{-4} \times 2.075071373^{3}}}{10^{-6}} \\
& =917317.5
\end{aligned}
$$

4. Finally, according to Eq. (28), the Chezy's resistance coefficient $C$ is:

$$
\begin{aligned}
& C=-5.343 \sqrt{g} \log \left(\frac{\varepsilon / \bar{D}}{4.75 \varphi(\eta)}+\frac{8.5}{\overline{R_{e f}}[\varphi(\eta)]^{3 / 2}}\right) \\
& =-5.343 \times \sqrt{9.81} \times \\
& \log \left(\frac{10^{-4} / 2.304526164}{4.75 \times 1.110576819}+\frac{8.5}{917317.5 \times 1.110576819^{3 / 2}}\right) \\
& =79.79486326 \mathrm{~m}^{0.5} / \mathrm{s} \cong 79.8 \mathrm{~m}^{0.5} / \mathrm{s}
\end{aligned}
$$

\subsection{Simplified Method}

In what follows, a simplified method is proposed for fast calculation of Chezy's coefficient $C$ with a reduced number of data. Neither the diameter $D$ of the conduit, nor the filling rate $\eta$ is required. Only four parameters are needed to evaluate Chezy's coefficient namely, the discharge $Q$, the slope $S$, the absolute roughness $\varepsilon$ and the kinematic viscosity $v$. All these parameters are easily measurable in practice. This simplified method, also based on the theory of the rough model, causes a maximum relative deviation of about $1.25 \%$ compared to the method described in section 4.1. This relative deviation is less than the relative error with which the absolute roughness is measured in practice. Assuming $\eta \neq \bar{\eta}$ and applying Eq. (31) for the rough model leads to:

$Q^{*}=\sqrt{2} \sigma(\bar{\eta})[\varphi(\bar{\eta})]^{3 / 2}$

Where $Q^{*}$ is the relative conductivity expressed as:

$Q^{*}=Q / \sqrt{g S D^{-5}}$ 
Consider a referential rough model having a diameter $\bar{D}$ equal to that of the full-model state corresponding to $\bar{\eta}=1$; Eq. (7) and Eq. (8) give respectively $\sigma(\bar{\eta})=\pi$ and $\varphi(\bar{\eta})=1$. As a result, Eq. (32) leads to $Q^{*}=\pi \sqrt{2}$. For this value of the relative conductivity, Eq. (32) indicates a second value of the filling rate equal to $\eta \approx 0.852$. We thus obtain a rough model with a diameter equal to that of the full-model state, characterized by the filling rate $\eta \approx 0.852$.

Consequently, the wetted perimeter $\bar{P}$ and the hydraulic radius $R_{h}$ are given by Eq. (9) and Eq. (11) respectively as:

$\bar{P}=2.352 \bar{D}$
$\overline{R_{h}}=0.3031 \bar{D}$

The diameter $\bar{D}$ of the full rough model is obtained for the relative conductivity $Q^{*}=\pi \sqrt{2}$, implying what follows:

$\bar{D}=(\pi \sqrt{2})^{-0.4}\left(\frac{Q}{\sqrt{g S}}\right)^{0.4}$

The calculation of Chezy's coefficient is readily carried out using the following steps: (36).

1. Compute the diameter $\bar{D}$ of the full model using Eq.

2. Compute then the wetted perimeter $\bar{P}$ and the hydraulic radius $R_{h}$ by the use of Eq. (34) and Eq. (35) respectively.

3. The Reynolds number $\overline{R_{e}}=4 Q /(\bar{P} v)$ in the rough model is then worked out.

4. With the computed values of $\overline{R_{h}}$ and $\overline{R_{e}}$, the nondimensional correction factor $\psi$ is explicitly determined using Eq. (24).

5. Finally, Chezy's coefficient $C$ is directly deduced from Eq. (25).

\subsection{Example 2}

Let us consider the data of example 1 to compute Chezy's resistance coefficient using the simplified method.

$Q=0.987 \mathrm{~m}^{3} / \mathrm{s}, S=3 \times 10^{-4}, \varepsilon=10^{-4} \mathrm{~m}, v=10^{-6} \mathrm{~m}^{2} / \mathrm{s}$.

1. According to Eq. (36), the diameter $\bar{D}$ of the full rough model is:

$$
\bar{D}=(\pi \sqrt{2})^{-0.4}\left(\frac{Q}{\sqrt{g S}}\right)^{0.4}
$$

$$
=(\pi \times \sqrt{2})^{-0.4}\left(\frac{0.987}{\sqrt{9.81 \times 3 \times 10^{-4}}}\right)^{0.4}=1.75753046 \mathrm{~m}
$$

2. Using Eq. (34) and Eq. (35), the wetted perimeter $\bar{P}$ and the hydraulic radius $\overline{R_{h}}$ are respectively:

$$
\begin{aligned}
& \bar{P}=2.352 \bar{D}=2.352 \times 1.75753046=4.13371164 \mathrm{~m} \\
& \overline{R_{h}}=0.3031 \bar{D}=0.3031 \times 1.75753046=0.53270748 \mathrm{~m}
\end{aligned}
$$

3. The Reynolds number $\bar{R}$ in the rough model is:

$$
\begin{aligned}
& \overline{R_{e}}=4 Q /(\bar{P} v)=4 \times 0.987 /\left(4.13371164 \times 10^{-6}\right) \\
& =955073.876
\end{aligned}
$$

4. According to Eq. (24), the non-dimensional correction factor $\psi$ is then:

$$
\begin{aligned}
& \psi=1.35\left[-\log \left(\frac{\varepsilon / \overline{R_{h}}}{19}+\frac{8.5}{\overline{R_{e}}}\right)\right]^{-2 / 5} \\
& =1.35 \times\left[-\log \left(\frac{10^{-4} / 0.53270748}{19}+\frac{8.5}{955073.876}\right)\right]^{-2 / 5} \\
& =0.72531217
\end{aligned}
$$

5. Using Eq. (25), the required value of Chezy's coefficient is:

$$
C=\frac{8 \sqrt{2 g}}{\psi^{5 / 2}}=\frac{8 \times \sqrt{2 \times 9.81}}{0.72531217^{5 / 2}}=79.1 \mathrm{~m}^{0.5} / \mathrm{s}
$$

Thus, comparing this result with that obtained in example 1 , we can observe that the relative deviation is less than $0.9 \%$ only.

\section{MAXIMUM OF CHEZY'S COEFFICIENT}

\subsection{General Relationship}

According to Eq. (20), the Chezy's resistance coefficient $C$ depends on three dimensionless variables namely, the relative roughness $\varepsilon / D$, the filling rate $\eta$ of the conduit and the Reynolds number $R_{e f}$. Its graphical representation is not easy, but it can be shown, as an indication, its variation for a fixed value of the relative roughness $\varepsilon / D$. This has been performed for different values of $\varepsilon / D$ and for Reynolds number $R_{e f}$ varying between $10^{4}$ and $10^{7}$.

Among all the obtained graphs, those of Figs. (1 and 2) are representative. Fig. (2) translates the variation of $C / \sqrt{g}$ versus the filling rate $\eta$ and the Reynolds number $R_{e f}$, for the value $\varepsilon / D=0$ corresponding to a smooth 


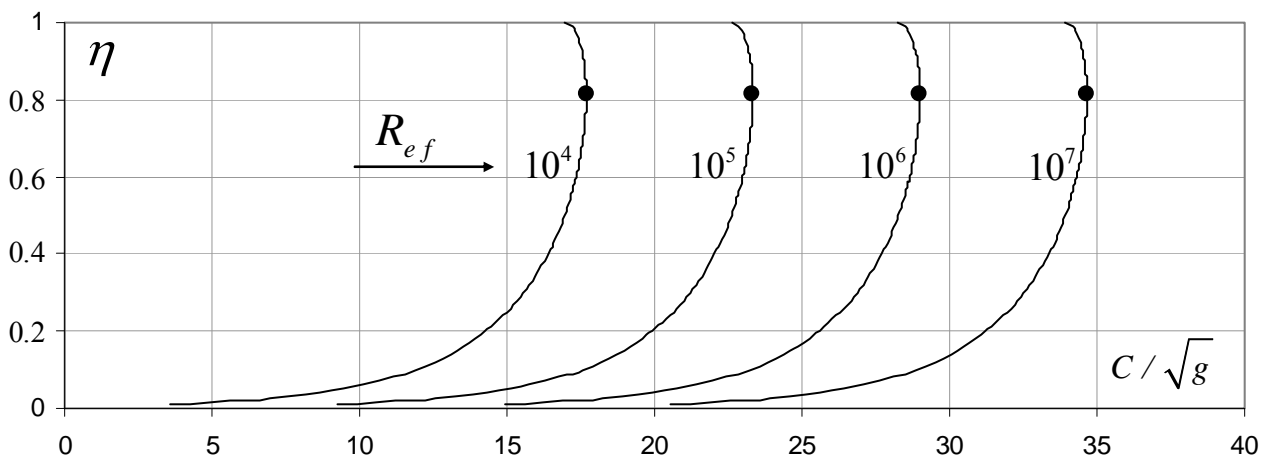

Fig. (2). Variation of $C / \sqrt{g}$ versus $\eta$ and $R_{e f}$ according to Eq. (20), for $\varepsilon / D=0$. (•) Maximum value $C_{\text {max. }} / \sqrt{g}$ obtained for $\eta \cong 0.8128$.

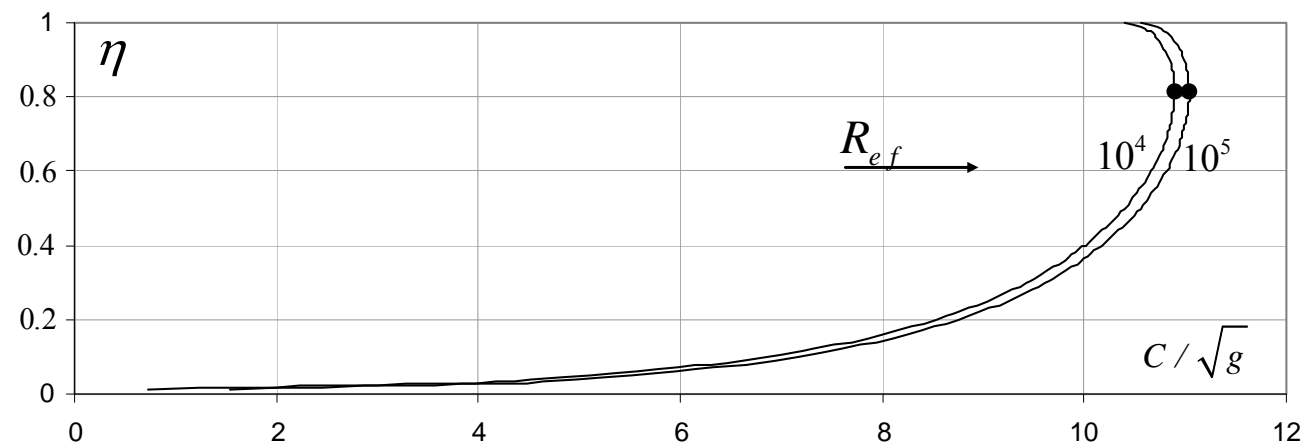

Fig. (3). Variation of $C / \sqrt{g}$ versus $\eta$ and $R_{e f}$ according to Eq. (20), for $\varepsilon / D=0.05$. (•) Maximum value $C_{\max .} / \sqrt{g}$ obtained for $\eta \cong 0.8128$.

inner wall of the conduit. Fig. (3) shows the variation of $C / \sqrt{g}$ versus the filling rate $\eta$ and the Reynolds number $R_{e f}$, for the value $\varepsilon / D=0.05$ corresponding to a state of the rough inner wall of the conduit. The chosen values of the relative roughness $\varepsilon / D$ correspond in fact to the extreme values of the Moody diagram.

Fig. (2) clearly shows that, for a given value of the Reynolds number $R_{e f}, C / \sqrt{g}$ increases with the increase of the filling rate $\eta$ up to a maximum value represented by the full sign on the figure. Beyond this maximum value, $C / \sqrt{g}$ decreases with the increase of the filling rate $\eta$ and the decrease continues until the full state of the conduit corresponding to $\eta=1$.

It should also be noted that, whatever the value of $R_{e f}$, the change in $C / \sqrt{g}$ according to $\eta$ is carried out rapidly at first, and undergoes a slight variation in a second time. The rapid variation of $C / \sqrt{g}$ is observed for a narrow range of $\eta$ that can be defined as $0 \leq \eta \leq 0.2$. Beyond the value $\eta=0.2, C / \sqrt{g}$ undergoes a very slow change in a wide range of $\eta$ independently of the value of the Reynolds number $R_{e f}$. This state of change can also be seen in Fig. (2). It also indicates that for the high chosen roughness value $(\varepsilon / D=0.05)$, the variation curves of $C / \sqrt{g}$ versus $\eta$ are very close to each other and merge for the values $R_{e f}>10^{5}$. This highlights the rough state of the flow, where $C / \sqrt{g}$ is almost independent of the Reynolds number $R_{e f}$ and depends solely on the value of the filling rate $\eta$ of the conduit.

The calculation reveals that Chezy's coefficient is the same for the particular values $\eta=0.5$ and $\eta=1$. In the range $0.5 \leq \eta \leq 1$, there are two normal depths for the same value of $C$ which is however very close to the maximum value $C_{\text {max. }}$ due to the low variation of the curve.

The most significant result obtained when plotting the variation of $C / \sqrt{g}$ as a function of $\eta$ and the Reynolds number $R_{e f}$, lies in the fact that the maximum value is achieved for the filling rate $\eta \cong 0.8128$, whatever the value of the relative roughness $\varepsilon / D$ and that of the Reynolds number $R_{e f}$. In other words, the maximum value of 
$C / \sqrt{g}$, and thus $C_{\text {max. }}$, is achieved at normal depth $y_{n} \cong 0,813 D$.

For $\eta=0,8128$, the function $\varphi(\eta)$ defined by Eq. (8) takes the following value:

$$
\begin{aligned}
& \varphi(\eta)=1-\frac{2 \times(1-2 \times 0.8128) \times \sqrt{0.8128 \times(1-0.8128)}}{\cos ^{-1}(1-2 \times 0.8128)} \\
& =1.217233628 \cong 1.21723
\end{aligned}
$$

Inserting this value in Eq. (20), leads to:

$$
\frac{C_{\text {max. }}}{\sqrt{g}}=-4 \sqrt{2} \log \left(\frac{\varepsilon / D}{4.504}+\frac{7.476}{R_{e f}}\right)
$$

or:

$$
C_{\text {max. }}=-4 \sqrt{2 g} \log \left(\frac{\varepsilon / D}{4.504}+\frac{7.476}{R_{e f}}\right)
$$

when $\varepsilon / D$ and $R_{e f}$ are the known parameters of the problem, Eq. (38) permits a direct determination of the maximum Chezy's resistance coefficient. When the diameter $D$ of the conduit is not given, the determination of the maximum of Chezy's resistance coefficient is possible by the use of Eq. (28), in which $\varphi(\eta)=1.217233628$. Hence:

$$
C_{\text {max. }}=-5.343 \sqrt{g} \log \left(\frac{\varepsilon / \bar{D}}{5.782}+\frac{6.329}{\overline{R_{e f}}}\right)
$$

According to Eq. (39), the maximum of Chezy's resistance coefficient $C_{\text {max. }}$ is related to the known parameters of the flow in the rough model, which can be then calculated in a simple manner even if the conduit diameter $D$ is not given. The following examples show the steps for calculating the maximum of Chezy's resistance coefficient.

\subsection{Example 3}

Compute the maximum value of Chezy's coefficient for the following data:

$$
D=1.5 \mathrm{~m} ; \varepsilon=10^{-4} \mathrm{~m} ; S=3 \times 10^{-4} ; v=10^{-6} \mathrm{~m}^{2} / \mathrm{s}
$$

1. According to Eq. (16), the Reynolds number $R_{e f}$ for the full state of the conduit is then:

$$
R_{e f}=4 \sqrt{2} \frac{\sqrt{g S D^{3}}}{v}=4 \times \sqrt{2} \times \frac{\sqrt{9.81 \times 3 \times 10^{-4} \times 1.5^{3}}}{10^{-6}}
$$

$$
=563776.551
$$

2. Finally, applying Eq. (38), one may obtain:

$$
\begin{aligned}
& C_{\text {max. }}=-4 \sqrt{2 g} \log \left(\frac{\varepsilon / D}{4.504}+\frac{7.476}{R_{e f}}\right) \\
& -4 \times \sqrt{2 \times 9.81} \times \log \left(\frac{10^{-4} / 1.5}{4.504}+\frac{7.476}{563776.551}\right) \\
& =80.65 \mathrm{~m}^{0.5} / \mathrm{s}
\end{aligned}
$$

\subsection{Example 4}

Compute the maximum value of Chezy's resistance coefficient in a circular conduit for the following data:

$$
Q=0.942 \mathrm{~m}^{3} / \mathrm{s}, S=4 \times 10^{-4}, \eta=0.65, \varepsilon=10^{-4} \mathrm{~m},
$$
$v=10^{-6} \mathrm{~m}^{2} / \mathrm{s}$

1. According to Eq. (7) and Eq. (8), $\sigma(\eta)$ and $\varphi(\eta)$ are respectively :

$\sigma(\eta)=\cos ^{-1}(1-2 \eta)=\cos ^{-1}(1-2 \times 0.65)$

$=1.87548898$

$\varphi(\eta)=1-\frac{2(1-2 \eta) \sqrt{\eta(1-\eta)}}{\cos ^{-1}(1-2 \eta)}$

$=1-\frac{2 \times(1-2 \times 0.65) \times \sqrt{0.65 \times(1-0.65)}}{\cos ^{-1}(1-2 \times 0.65)}$

$=1.15259048$

2. Eq. (31) gives the diameter $\bar{D}$ of the rough model as:

$\bar{D}=[\sqrt{2} \sigma(\eta)]^{-0.4}[\varphi(\eta)]^{-0.6}\left(\frac{Q}{\sqrt{g S}}\right)^{0.4}$
$=[\sqrt{2} \times 1.87548898]^{-0.4} \times 1.15259048^{-0.6}$

$\times\left(\frac{0.942}{\sqrt{9.81 \times 4 \times 10^{-4}}}\right)^{0.4}=1.838305722 \mathrm{~m}$

3. According to Eq. (29), the Reynolds number $\overline{R_{e f}}$ for the full state of the rough model is then :

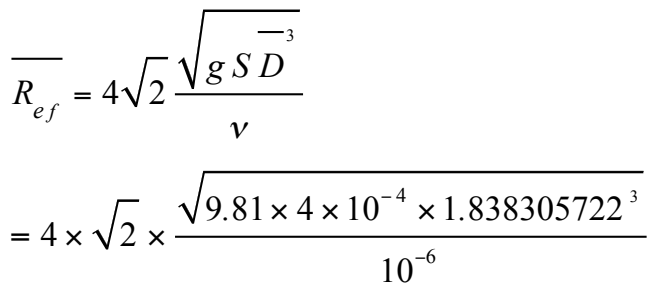

$=883214.31$ 
4. As a result, Eq. (39) leads to:

$$
\begin{aligned}
& C_{\text {max. }}=-5.343 \sqrt{g} \log \left(\frac{\varepsilon / \bar{D}}{5.782}+\frac{6.329}{\overline{R_{e f}}}\right) \\
& =-5.343 \times \sqrt{9.81} \times \log \left(\frac{10^{-4} / 1.838305722}{5.782}+\frac{6.329}{883214.31}\right) \\
& =80 \mathrm{~m}^{0.5} / \mathrm{s}
\end{aligned}
$$

\section{CONCLUSION}

Using the general discharge relationship, the expression of Chezy's coefficient $C$ was established for a circular conduit. The obtained expression clearly showed that $C$ depends on the relative roughness $\varepsilon / D$, the filling rate $\eta$ of the conduit and the Reynolds number $R_{e f}$ characterizing the full state of the flow. This in turn depends on the slope $S$, the diameter $D$ of the conduit and the kinematic viscosity $v$. All parameters influencing the flow are represented in the expression of $C$, unlike current relationships. When all these parameters are given, the resulting expression is used to calculate explicitly the required value of $C$. When the diameter $D$ of the conduit is not a given data of the problem, the explicit calculation of $C$ is still possible through the use of the rough model method. $C$ is then expressed as a function of the known parameters of the flow in the rough model. In this case, the calculation of $C$ requires the discharge $Q$, the slope $S$, the absolute roughness $\varepsilon$, the filling rate $\eta$ and the kinematic viscosity $v$. When the user does not have all the data of the problem, the explicit calculation of $C$ is still possible thanks to the simplified method that was clearly described. This method uses the minimum measurable data in practice which are the discharge $Q$, the slope $S$, the absolute roughness $\varepsilon$ and kinematic viscosity $v$. This simplified method gives very satisfactory results.

The paper was completed by the particular study of the coefficient $C$. The graphical representation showed a rapid increase in the range $0<\eta \leq 0.2$. It also showed a slight increase in $C$ beyond $\eta=0.2$ and then reached a maximum at the filling rate $\eta=0.8128$. $C$ then undergoes a slight diminution to the full state of the conduit corresponding to $\eta=1$.

\section{ABBREVIATIONS}

$$
\begin{aligned}
& A=\text { Water area } \\
& C=\text { Chezy's coefficient } \\
& D=\text { Diameter of the conduit } \\
& \bar{D}=\text { Diameter of the rough model } \\
& D_{h}=\text { Hydraulic diameter } \\
& f=\text { Friction factor } \\
& g=\text { Acceleration due to gravity } \\
& S=\text { Slope of the conduit } \\
& P=\text { Wetted perimeter }
\end{aligned}
$$

$Q=$ Discharge

$Q^{*}=$ Relative conductivity

$R_{\mathrm{e}}=$ Reynolds number

$R_{e f}=$ Reynolds number at the full state of the conduit

$R_{h}=$ Hydraulic radius

$y_{n}=$ Normal depth

$\varepsilon=$ Absolute roughness

$\eta=$ Filling rate equal to $y_{n} / D$

$\psi=$ Non-dimensional correction factor

$v=$ kinematic viscosity

\section{CONFLICT OF INTEREST}

The author confirms that this article content has no conflict of interest.

\section{ACKNOWLEDGEMENTS}

Declared none.

\section{REFERENCES}

[1] E. Ganguillet, and W.R Kutter, "An investigation to establish a new general formula for uniform flow of water in canals and rivers", Zeitschrift des Oesterreichischen Ingenieur und Architekten Vereines, vol. 21, no. 1, pp. 6-25, no.2-3, pp. 46-59, 1869.

[2] H. Bazin, "Etude d'une nouvelle formule pour calculer le débit des canaux découverts", Annales des ponts et chausses, vol. 14, ser.7, 4ème trimestre, pp. 20-70, 1897.

[3] R.W. Powell, "Resistance to flow in rough channels", Trans. Am. Geophys. Union, vol. 31, no. 4, pp. 575-582, 1950.

[4] V.T. Chow, Ed., Open-Channel Hydraulics. McGraw Hill: New York, 1973.

[5] P. K. Swamee, and P.N. Rathie, "Exact solutions for normal depth problem", J. Hydraul. Res., vol. 42, no. 5, pp. 541-547, 2004.

[6] V.L. Streeter, "Frictional resistance in artificially roughened pipes", Trans. ASCE, vol. 101, pp. 681-704, 1936.

[7] S.A. Ead, N. Rajaratnam, C. Katopodis, and F. Ade, "Turbulent open-channel flow in circular corrugated culverts", J. Hydraul. Eng. vol. 126, no. 10, pp. 750-757, 2000.

[8] R. Pyle, and P. Novak, "Coefficient of friction in conduits with large roughness", J. Hydraul. Res., vol. 19, no. 2, pp. 119-140, 1981.

[9] V. Marone, "Le resistenze al movimento uniforme in un alveo chiuso o aperto di sezione rettangolare e scabrezza definita", L'Energia Elettrica, vol. 1, pp. 1-20, 1970.

[10] A.E. Perry, W.H. Schofield, and P.N. Joubert, "Rough wall turbulent boundary layers", J. Fluid Mech., vol. 37, no. 2, pp. 383-413, 1969.

[11] D. Naot, I. Nezu, and H. Nakagawa, "Hydrodynamic Behaviour of Partly Vegetated Open Channels", J. Hydraul. Eng., vol. 122, no. 11, pp. 625-633, 1996.

[12] O. Giustolisi, "Using genetic programming to determine Chezy resistance coefficient in corrugated channels", J. Hydroinf., vol. 6, no. 3, pp. 157-173, 2004.

[13] B. Achour, and S. Sehtal, "The rough model method (RMM). application to the computation of normal depth in circular conduit", Open Civil Eng. J., vol. 8, pp. 57-63, 2014.

[14] B. Achour, and M. Riabi, "Design of a pressurized trapezoidal shaped conduit using the rough model method (Part 1)", Adv. Mater. Res., vols. 945-949, pp. 892-898, 2014.

[15] B. Achour, "Computation of normal depth in trapezoidal open channel using the rough model method", Adv. Mater. Res., vol. 955-959, pp. 3231-3237, 2014. 
[16] M. Riabi, and B. Achour, "Design of a pressurized circular pipe with benches using the rough model method (RMM)", Adv. Mater. Res., vol. 960-961, pp. 586-591, 2014.

[17] B. Achour, and A. Bedjaoui, "Design of a pressurized trapezoidal shaped conduit using the rough model method (part 2)", Appl. Mech. Mater., vol. 580-583, pp. 1828-1841, 2014.

[18] B. Achour, "Computation of normal depth in horseshoe shaped tunnel using the rough model method", Adv. Mater. Res., vol. 1006-1007, pp. 826-832, 2014.

[19] B. Achour, "Design of a pressurized rectangular conduit with triangular bottom using the rough model method", Open Civil Eng. J., vol. 8, pp. 205-212, 2014.

[20] B. Achour, "Computation of normal depth in parabolic cross sections using the rough model method", Open Civil Eng. J., vol. 8, pp. 213-218, 2014.
[21] B. Achour, "Design of a pressurized rectangular-shaped conduit using the rough model method", Appl. Mech. Mater., vol. 641-642, pp. 261-266, 2014.

[22] B. Achour, and M. Khattaoui, "Design of pressurized vaulted rectangular conduits using the rough model method (part 2)", $A d v$. Mater. Res., vol. 1025-1026, pp. 24-31, 2014.

[23] B. Achour, and A. Bedjaoui, "Discussion. exact solutions for normal depth problem", J. Hydraul. Res., vol. 44, no. 5, pp. 715-717, 2006.

[24] B. Achour, and A. Bedjaoui, "Turbulent pipe-flow computation using the rough model method (RMM)", J. Civil Eng. Sci., vol. 1, no. 1, pp. 36-41, 2012.

[25] B. Achour, "Design of pressurized vaulted rectangular conduits using the rough model method", Adv. Mater. Res., vol. 779-780, pp. 414-419, 2013.

Received: December 12, 2014

Revised: March 17, 2015

Accepted: March 21, 2015

(C) Bachir Achour; Licensee Bentham Open.

This is an open access article licensed under the terms of the Creative Commons Attribution Non-Commercial License (http://creativecommons.org/licenses/ by-nc/3.0/) which permits unrestricted, non-commercial use, distribution and reproduction in any medium, provided the work is properly cited. 